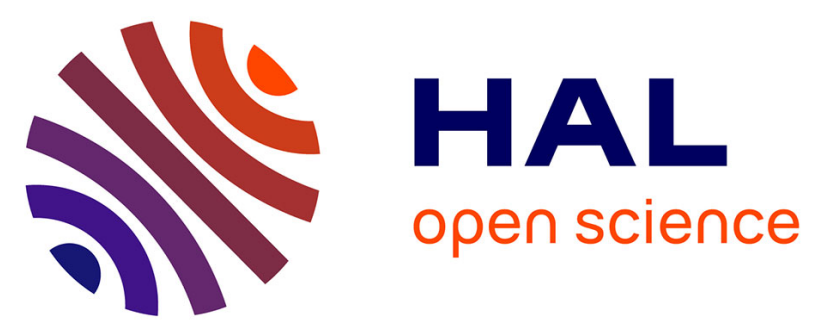

\title{
Quantification of stiffness measurement errors in resonant ultrasound spectroscopy of human cortical bone
}

\author{
Xiran Cai, Laura Peralta, Pierre-Jean Gouttenoire, Cécile Olivier, Françoise
}

Peyrin, Pascal Laugier, Quentin Grimal

\section{- To cite this version:}

Xiran Cai, Laura Peralta, Pierre-Jean Gouttenoire, Cécile Olivier, Françoise Peyrin, et al.. Quantification of stiffness measurement errors in resonant ultrasound spectroscopy of human cortical bone. Journal of the Acoustical Society of America, 2017, 142 (5), pp.2755-2765. 10.1121/1.5009453 . hal01701981

\section{HAL Id: hal-01701981 \\ https: / hal.sorbonne-universite.fr/hal-01701981}

Submitted on 6 Feb 2018

HAL is a multi-disciplinary open access archive for the deposit and dissemination of scientific research documents, whether they are published or not. The documents may come from teaching and research institutions in France or abroad, or from public or private research centers.
L'archive ouverte pluridisciplinaire HAL, est destinée au dépôt et à la diffusion de documents scientifiques de niveau recherche, publiés ou non, émanant des établissements d'enseignement et de recherche français ou étrangers, des laboratoires publics ou privés. 
${ }_{1}$ Quantification of stiffness measurement errors in resonant ultrasound spectroscopy 2 of human cortical bone

3 Xiran Cai, ${ }_{3}^{1, a)}$ Laura Peralta, ${ }^{1}$ Pierre-Jean Gouttenoire ${ }^{2}$ Cécile Olivier, ${ }^{3, \text { b) }}$ Françoise ${ }_{4} \quad$ Peyrin, ${ }^{3,}$ b) Pascal Laugier, ${ }^{1}$ and Quentin Grimal ${ }^{1}$

${ }_{5}{ }^{1)}$ Sorbonne Universités, UPMC Univ Paris 06, INSERM UMR-S 1146,

$6 \quad$ CNRS UMR 7371, Laboratoire d'Imagerie Biomédicale,

$7 \quad 15$ rue de l'Ecole de Médecine, Paris 75006, France

2) ESRF, 71 Avenue des Martyrs, Grenoble 38043, France

3) Univ. Lyon, INSA-Lyon, Université Claude Bernard Lyon 1, CNRS, INSERM, CREATIS UMR 5220, U1206, 7 Avenue Jean Capelle, Villeurbanne 69621, France

(Dated: October 6, 2017) 
Measurement errors in resonant ultrasound spectroscopy

Resonant ultrasound spectroscopy (RUS) is the state-of-the-art method used to investigate the elastic properties of anisotropic solids. Recently, RUS was applied to measure human cortical bone, an anisotropic material with low Q-factor (20), which is challenging due to the difficulty in retrieving resonant frequencies. Determining the precision of the estimated stiffness constants is not straightforward because RUS is an indirect method involving minimizing the distance between measured and calculated resonant frequencies using a model. This work was motivated by the need to quantify the errors on stiffness constants due to different error sources in RUS, including uncertainties on the resonant frequencies and specimen dimensions and imperfect rectangular parallelepiped (RP) specimen geometry. The errors were firstly investigated using Monte-Carlo simulations with typical uncertainty values of experimentally measured resonant frequencies and dimensions assuming a perfect RP geometry. Secondly, the exact specimen geometry of a set of bone specimens were recorded by synchrotron radiation micro-computed tomography. Then, a 'virtual' RUS experiment is proposed to quantify the errors induced by imperfect geometry. Results show that for a bone specimen of $\sim 1^{\circ}$ perpendicularity and parallelism errors, an accuracy of a few percent $(<6.2 \%)$ for all the stiffness constants and engineering moduli is achievable. PACS numbers: PACS: XXXX

a) Electronic mail: xiran.cai@upmc.fr; Corresponding author.

b) Also at: ESRF, 71 Avenue des Martyrs, Grenoble 38043, France 
Measurement errors in resonant ultrasound spectroscopy

\section{${ }_{14}$ I. INTRODUCTION}

15 Bone adaptation in response to mechanical loading and the subsequent optimization of 16 bone strength are regulated by mechanosensitive osteocytes, which are capable of sensing ${ }_{17}$ strain $^{1}$. For a given load, bone stiffness determines the local strain, hence investigating bone 18 stiffness in detail should allow gaining insight into bone functional adaptation mechanisms 19 and bone strength.

20 As the structure of human cortical bone, like many natural materials, is hierarchical ${ }^{2}$, it ${ }_{21}$ is necessary to investigate it at different scales. In particular, cortical bone elastic properties 22 at the mesoscale (millimeter-scale) are of special interest as they depend on tissue properties 23 at all the smaller length scales and have a direct impact on the mechanical behavior of bone 24 at the macroscale ${ }^{3,4}$. In addition, this is the level at which cortical bone functions, in concert 25 with the overall gross shape of a bone in resisting functional loads ${ }^{5}$. The mesoscopic level 26 is also appropriate to investigate the regional variations of the elastic properties within a ${ }_{27}$ bone $^{6}$, which is necessary to refine finite element models to predict patterns of stress and 28 strain. In this context, precise and practical measurement methods for assessing cortical 29 bone elasticity at the mesoscale are needed.

30 In general, bone material can be considered as a transversely isotropic or orthotropic 31 material, hence engineering moduli such as Young's moduli, shear moduli, and Poisson's 32 ratio can be derived from the components of the stiffness tensor. Ultrasonic techniques зз are well suited to probe the anisotropic elastic properties of bone. The most widely used 34 ultrasonic measurement method, which was introduced by Lang ${ }^{7}$ and used by many research 35 groups $^{8-14}$, consists in measuring the ultrasonic wave velocity (UWV). Despite its apparent 36 simplicity, UWV measurements present several pitfalls that must be carefully considered. ${ }_{37}$ The final result can be affected by some factors, including the size of the measured specimen 38 compared to the wavelength, the presence of heterogeneities, or the signal processing required 39 to estimate the time of flight to calculate velocity ${ }^{15,16}$.

40 Resonant ultrasound spectroscopy (RUS) has been recently introduced as an alternative ${ }_{41}$ technique to the measurement of human cortical bone stiffness ${ }^{17}$. RUS has been extensively 42 used since 1990's to investigate the elastic properties of solids as diverse as piezoelectric ${ }_{43}$ materials $^{18}$, metallic alloys ${ }^{19}$, metallic glasses ${ }^{20}$ and composites $^{21}$, hard polymers ${ }^{22}$, wood ${ }^{23}$, 44 and mineralized tissues ${ }^{17,24,25}$ for applications ranging from theoretical physics to industrial 
Measurement errors in resonant ultrasound spectroscopy

45 problems. The main advantage of RUS, compared to other techniques such as UWV mea46 surements and mechanical testing, is that the full set of the elastic tensor can be assessed ${ }_{47}$ non-destructively from a single measurement ${ }^{26,27}$. Briefly, in a RUS experiment, resonant ${ }_{48}$ frequencies of a free vibrating specimen are retrieved from the resonant spectrum measured 49 by a pair of ultrasonic transducers. Then, the stiffness constants are adjusted using an 50 iterative numerical procedure (inverse problem) until the calculated eigenfrequencies of a ${ }_{51}$ free vibration object (forward problem) match with the experimentally measured resonant 52 frequencies.

${ }_{53}$ Determining the precision of the different stiffness constants measured by RUS is not ${ }_{54}$ straightforward because RUS is an indirect method to obtain stiffness constants, involving ${ }_{55}$ the minimization of the distance between measured and calculated frequencies. Essentially, ${ }_{56}$ elasticity estimation errors arise from two sources ${ }^{19,26}(1)$ the imperfectly measured resonant ${ }_{57}$ frequencies; and (2) inadequate geometry of the forward model. The latter is caused by ${ }_{58}$ possible shape imperfections (i.e., non perfectly parallel or perpendicular surfaces) not taken 59 into account in the model, and metrological errors in the measurement of the specimen's 60 dimensions.

${ }_{61}$ The effects of RUS measurement errors have been addressed to some extent in several 62 studies in the case of perfectly rectangular parallelepiped (RP) shaped specimen geome${ }_{63} \operatorname{try}^{26,28-30}$. Regarding the first source of error (imperfectly measured resonant frequencies), ${ }_{64}$ the uncertainties on the determined stiffness constants have been estimated using the per${ }_{65}$ turbation theory (assuming perfect RP specimen geometry). By determining the sensitivity ${ }_{66}$ of the resonant frequencies to the stiffness constants, the uncertainties of the stiffness con${ }_{67}$ stants can be quantified as a function of the relative root mean square error (RMSE) $\sigma_{f}$ ${ }_{68}$ expressing the misfit between the measured and calculated resonant frequencies ${ }^{26,29}$. For ${ }_{69}$ instance, Sedlack et al. ${ }^{30}$ quantified the typical uncertainties measured on a silicon carbide 70 ceramics parallelepiped specimen and found relative measurement errors of less than $0.35 \%$, ${ }_{71} 0.80 \%$ and $2.80 \%$ for shear, longitudinal and off-diagonal stiffness constants respectively, for ${ }_{72} \sigma_{f}=0.25 \%$. Regarding the second source of error (imperfect geometry), on an empirical 73 basis, Migliori et al. ${ }^{26,31}$ recommended that shape errors in parallelism and perpendicularity 74 between faces should be limited to $0.1 \%$ in order to keep errors on stiffness constants within 75 acceptable bounds, that is, close to $1 \%$. However, there is no data in the open literature to 76 support these numbers, as far as we know. 
Measurement errors in resonant ultrasound spectroscopy

77 When measuring bone elasticity using RUS, errors on the measured resonant frequencies 78 are larger compared to the case of other materials. This is related to the high viscoelastic 79 damping of the material (Q-factor $\sim 20$ ) resulting in resonant peaks overlapping and a 80 lower accuracy of the measured frequencies compared to the case of high-Q materials ${ }^{22,32}$. ${ }_{81}$ In many RUS applications only a few specimens are measured, and much time is devoted to 82 specimen's preparation in order to achieve an excellent geometrical quality. In contrast, the 83 high variability of elastic properties in biological materials, in particular within a bone ${ }^{33}$, ${ }_{84}$ implies that several tens of specimens should be measured in order to obtain representative ${ }_{85}$ values of stiffness. As a result, polishing each bone specimen in successive steps ${ }^{31}$ to obtain 86 a very high geometrical quality is not practicable. Hence, the question arises of the accuracy ${ }_{87}$ of the measured elasticity after a relatively simple preparation with a precision saw. To the s8 best of our knowledge, no systematic study has been conducted about neither the effects 89 of an imperfect specimen geometry on the elastic properties of cortical bone measured by ${ }_{90}$ RUS, nor the combined effects when resonant frequencies uncertainties are also considered. ${ }_{91}$ The objective of this study is to quantify the experimental errors when measuring cortical 92 bone elasticity with RUS. We take advantage of recent advances in RUS inverse problem 93 to quantify sources of errors using Monte Carlo simulations. Namely, the step consisting in ${ }_{94}$ pairing measured frequencies and their calculated counterparts in the forward problem, pre95 viously achieved by an expert user with a trial-and-error method, was recently automated ${ }^{34}$. 96 This allows an automated processing of RUS spectra which is a necessary condition for ${ }_{97}$ Monte Carlo analyses of error propagation. The following error sources are considered: 98 (1) uncertainties on the measurement of frequencies; (2) uncertainties on the measurement 99 of dimensions (assuming a perfect RP shape); (3) imperfect specimen geometry (deviation 100 from a perfect RP). Although our primary focus is the application of RUS to measure bone, 101 the methodology introduced in this work and the quantified errors are of general interest for 102 the discussion of the precision and accuracy of RUS measurements of various materials.

${ }_{103}$ Section II briefly recalls the theory of RUS, then Section III presents the specimens in104 cluded in this study and their experimental measurements. Firstly, their elasticity is assessed 105 by RUS and secondly, the geometry of the specimens is obtained from synchrotron radiation 106 micro-computed tomography (SR- $\mu \mathrm{CT}$ ) images. In Section IV, the effects of measurement 107 uncertainties caused by both specimen dimensions and frequency errors, are investigated by 108 Monte Carlo simulations. Section V investigates the errors associated to the deviation of 
Measurement errors in resonant ultrasound spectroscopy

109 the specimens's shape from a perfect RP. Here, the finite element method (FEM) is used 110 to calculate resonant frequencies accounting for the actual shape of the specimen. Finally, ${ }_{111}$ results are discussed in Section VI.

\section{${ }_{112}$ II. RUS THEORY}

${ }_{113}$ RUS method is extensively described elsewhere ${ }^{26,27}$. Here we summarize the process as 114 implemented in the present work. The determination of stiffness constants of the material 115 constitutive of a specimen of RP shape consists of the following steps: (1) the resonant 116 frequencies $\mathbf{f}^{\text {exp }}$ of the specimen are measured; (2) using $\mathbf{f}^{\text {exp }}$, the stiffness constants $\mathbf{C}_{i j}$ ${ }_{117}(i j=11,33,13,44,66)$ are determined by solving an optimization problem, i.e., minimizing ${ }_{118}$ the objective function (Eq. (1) $)^{26}$ :

$$
F\left(\mathbf{C}_{i j}\right)=\sum_{k}\left(\frac{f_{k}^{e x p}-f_{k}^{\text {mod }}\left(\mathbf{C}_{i j}\right)}{f_{k}^{e x p}}\right)^{2}
$$

119 where $\mathbf{f}^{\text {mod }}$ are simulated eigenfrequencies of a model of the specimen (forward problem) 120 and $k$ is the index of the eigenfrequency. In the optimization, the mass is assumed known, 121 and the shape is assumed to be a perfect RP of known dimensions, collected in vector ${ }_{122} \mathbf{d i m}$. Frequencies $\mathbf{f}^{\text {mod }}$ are calculated with the Rayleigh-Ritz method (RRM), which is a 123 semi-analytical method that yields the result in a fraction of a second on a modern desktop 124 computer. In Eq. (1), the experimental and simulated frequencies are assumed to be paired. ${ }_{125}$ In the present work, pairing is done automatically in a Bayesian optimization strategy ${ }^{34}$.

\section{${ }_{126}$ III. MEASUREMENTS}

\section{${ }_{127}$ A. Specimens}

${ }_{128}$ Cortical bone specimens were harvested from the left femur of 18 human cadavers. The fe129 murs were provided by the Départment Universitaire d'Anatomie Rockefeller (Lyon, France) 130 through the French program on voluntary corpse donation to science. The tissue donors or 131 their legal guardians provided informed written consent to give their tissue for investiga132 tions, in accord with legal clauses stated in the French Code of Public Health. Among the 
Measurement errors in resonant ultrasound spectroscopy

13318 donors, 11 were females and 7 were males $(50-95$ years old, $77 \pm 12.3$, mean \pm SD). The 134 fresh material was frozen and stored at $-20^{\circ} \mathrm{C}$.

135 The samples were slowly thawed and then, for each femur, approximately a $10 \mathrm{~mm}$ thick 136 cross section was cut perpendicular to the bone axis from the mid-diaphysis. The cross 137 section was then cut into 4 pieces (Fig. 1a). Two of these pieces (lateral and medial) were ${ }_{138}$ then used to prepare a RP specimen. They were fixed on a stainless steel block (Fig. 1b) 139 that has three mutually perpendicular faces. Without unmounting the specimen, the steel 140 block was successively positioned on each of these three faces on a reference stage in order 141 to cut with a water-cooled low-speed diamond wire saw (Model 3241, Well, Lyon, France) 142 in three mutually perpendicular planes. From each donor, one or two RP shaped specimens 143 were prepared, which led to a set of 23 specimens. The nominal specimen size was $3 \times 4 \times 5$ ${ }_{144} \mathrm{~mm}^{3}$ in radial (axis 1), circumferential (axis 2) and axial direction (axis 3), respectively, 145 defined by the anatomic shape of the femoral diaphysis. All specimens were kept hydrated ${ }_{146}$ during sample preparation. The dimensions $\left(\mathbf{d i m}^{\exp }\right)$ and mass $\left(\mathrm{m}^{\exp }\right)$ of each specimen ${ }_{147}$ were measured by a digital caliper (precision $\pm 0.01 \mathrm{~mm}$ ) and a balance (precision \pm 0.1 $148 \mathrm{mg})$, respectively.

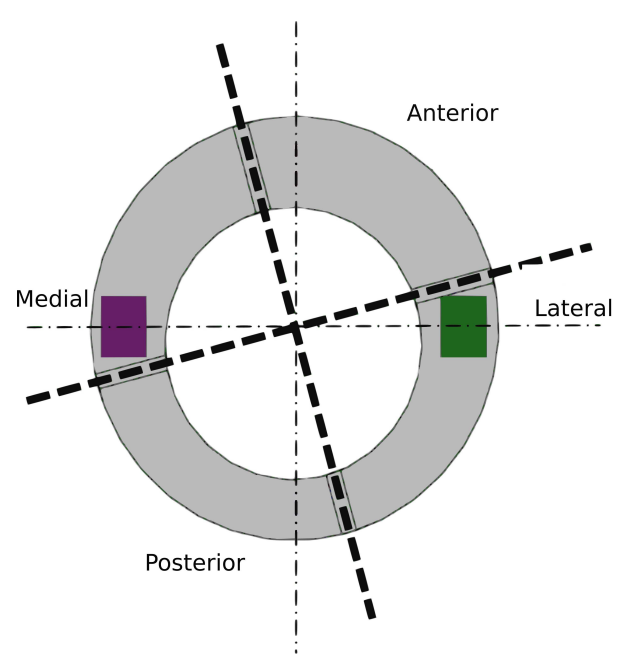

a)

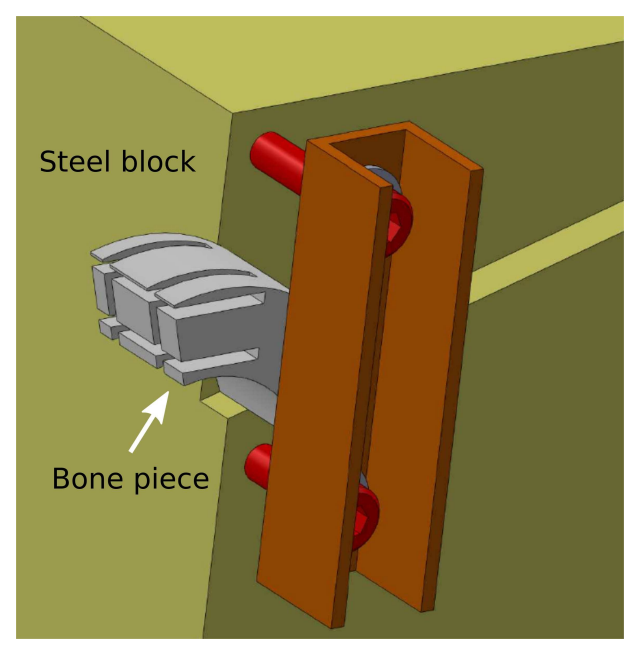

b)

Figure 1. a) the cross section of a femur was cut into 4 pieces according to the anatomical locations: lateral, medial, posterior and anterior; b) the steel block on which a bone piece was fixed for being cut by a diamond wire saw to retrieve a cuboid specimen. Two pairs of perpendicular cuts were realized by successively positioning the block on a reference stage with two mutually perpendicular faces. 
Measurement errors in resonant ultrasound spectroscopy

\section{${ }_{149}$ B. Bone elasticity measurements by RUS}

The experiments to measure the resonant frequencies and the numerical inversion to 151 calculate the stiffness constants were performed following the RUS methodology specially ${ }_{152}$ adapted for bone and extensively presented elsewhere ${ }^{17,34}$. The procedure is briefly described 153 as below. The bone specimen was placed on two opposite corners between two ultrasonic 154 transducer (V154RM, Panametrics, Waltham, MA), one for emission and one for reception, 155 to achieve a free boundary condition for vibration (Fig. 2).

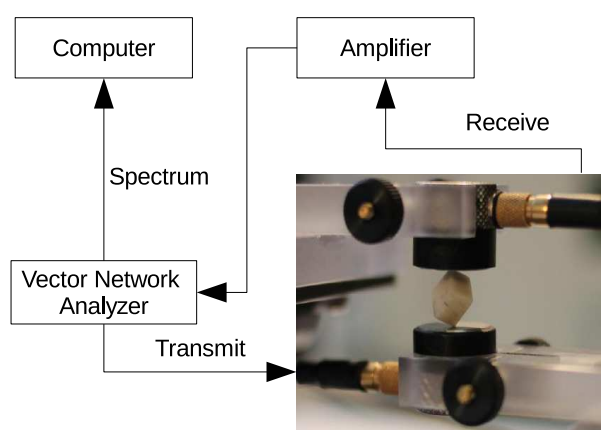

Figure 2. The RUS setup used in this study. A bone specimen is placed between two ultrasonic transducers at the two opposite corners to achieve a free boundary condition for vibration.

The frequency response of the vibration in a specified bandwidth, tuned so as to measure 157 the 20-30 first resonant frequencies, was amplified by a broadband charge amplifier (HQA${ }_{158} 15$ M-10T, Femto Messtechnik GmbH, Berlin, Germany) and then recorded by a vector 159 network analyzer (Bode 100, Omicron Electronics GmbH, Klaus, Austria). Six consecutive 160 spectrum acquisitions were performed on each specimen at different orientations in order 161 to maximize the number of detectable resonant frequencies. Then, the resonant frequencies ${ }_{162}$ were extracted from the spectra using the method dedicated to highly attenuative material ${ }^{32}$ 163 (Fig. 3).

${ }_{164}$ Finally, assuming a transversely isotropic symmetry ${ }^{12,35}$, the stiffness constants $\mathbf{C}_{i j}^{\exp }$, 165 were automatically calculated by solving the inverse problem formulated in a Bayesian 166 framework $^{34}$ (Sec. II). The prior information of the distribution of the stiffness constants, ${ }_{167}$ required for the Bayesian analysis, was taken from a previous study ${ }^{13}$. In the elastic tensor, ${ }_{168} C_{12}=C_{11}-2 C_{66}$ and $(1-2)$ is the isotropy plane; $C_{11}$ and $C_{33}$ are the longitudinal stiffness ${ }_{169}$ constants, $C_{12}$ and $C_{13}$ are the off-diagonal stiffness constants and $C_{44}$ and $C_{66}$ represent the 170 shear stiffness constants. 
Measurement errors in resonant ultrasound spectroscopy

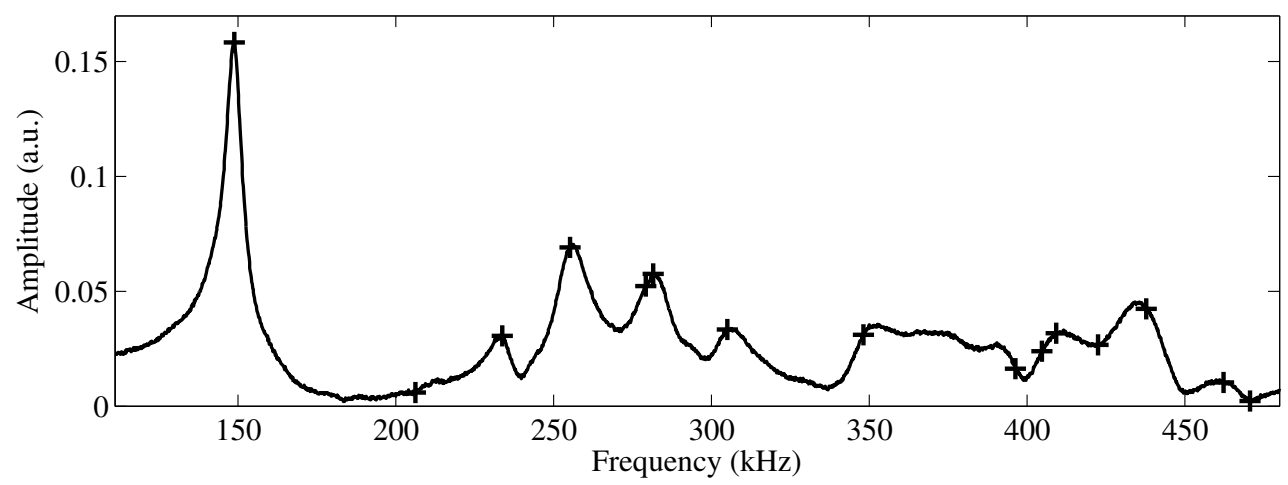

Figure 3. A typical resonant spectrum measured on a bone specimen. The plus signs $(+)$ represent the extracted resonant frequencies.

\section{${ }_{171}$ C. Specimen geometry}

172 The exact shape of the specimens and thus, deviation from the ideal RP shape was 173 obtained using SR- $\mu$ CT 3-D imaging, which was performed on the beamline ID19 at the ${ }_{174}$ European Synchrotron Radiation Facility (ESRF, Grenoble, France). This SR- $\mu$ CT setup 175 is based on a $3 \mathrm{D}$ parallel beam geometry acquisition ${ }^{36,37}$. The beam energy was tuned to $17626 \mathrm{keV}$ by using a (Si111) double crystal monochromator. A full set of 2D radiographic 177 images were recorded using a CDD detector (Gadox scintillator, optic lenses, $2048 \times 2048$ 178 Frelon Camera) by rotating the specimen in 1999 steps within a $360^{\circ}$ range of rotation. The 179 detector system was fixed to get a pixel size of $6.5 \mu \mathrm{m}$ in the recorded images in which a 180 region of interest of $1400 \times 940$ pixels was selected to fit the specimen.

${ }_{181}$ For each specimen, the SR- $\mu \mathrm{CT}$ image (Fig. 4a) was reconstructed and binarized to 182 get the bone phase. In RUS, the material of the measured specimen is considered as a 183 homogeneous material. Here, the specimen is much larger than the representative volume ${ }_{184}$ element of continuum mechanics ${ }^{4}$. Accordingly, the vascular pores that are visible in the 3D 185 image were filled up (Fig. 4b) using mathematical morphology operations to obtain a mask 186 of each slice. Then the convex envelope of the bone masks was calculated and considered to 187 be the exact shape of the specimen.

${ }_{188}$ The quality of the geometry of the specimen was analyzed based on the reconstructed SR${ }_{189} \mu \mathrm{CT}$ volume. The coordinates of the cloud of points of each specimen's face were collected 190 and the equation of the planes fitting each face in the least-square sense were determined. ${ }_{191}$ The angles $\alpha$ and $\beta$ between the normal of the planes were used to quantify the quality of 192 the specimen's geometry compared to a perfect RP (Fig. 5). The perpendicularity errors 


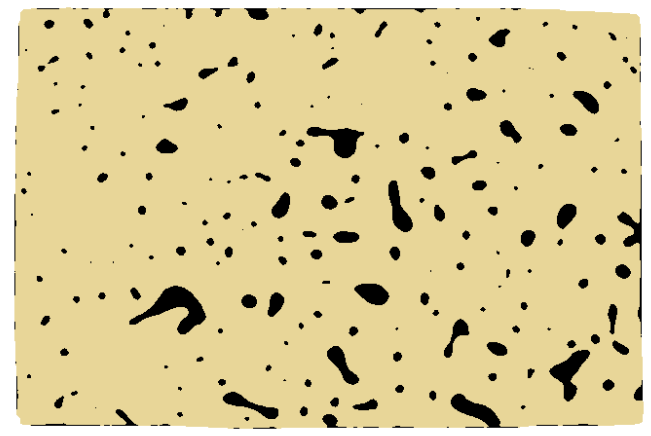

a)

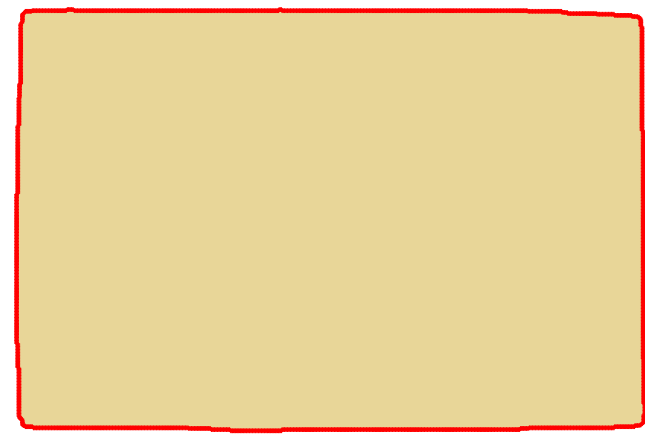

b)

Figure 4. a) A slice of the binarized image of bone structure. b) The mask of the bone slice after filling up the pores (the black parts in a)) and the contours (red color) detected from the mask. The contours of all the masks determine the external envelope of the specimen which was used to quantify its perpendicularity and parallelism quality.

193 between adjacent faces were quantified by $\delta \alpha=90^{\circ}-\alpha$. The parallelism errors between 194 opposite faces were quantified by $\delta \beta=180^{\circ}-\beta$. The values of the angle errors for the 23 195 specimens (12 $\delta \alpha$ and $3 \delta \beta$ per specimen) are collected in Fig. 6. The deviations (mean \pm std) 196 from ideal perpendicularity and parallelism were $-0.07^{\circ} \pm 0.85^{\circ}$ and $0.30^{\circ} \pm 0.78^{\circ}$, respectively.
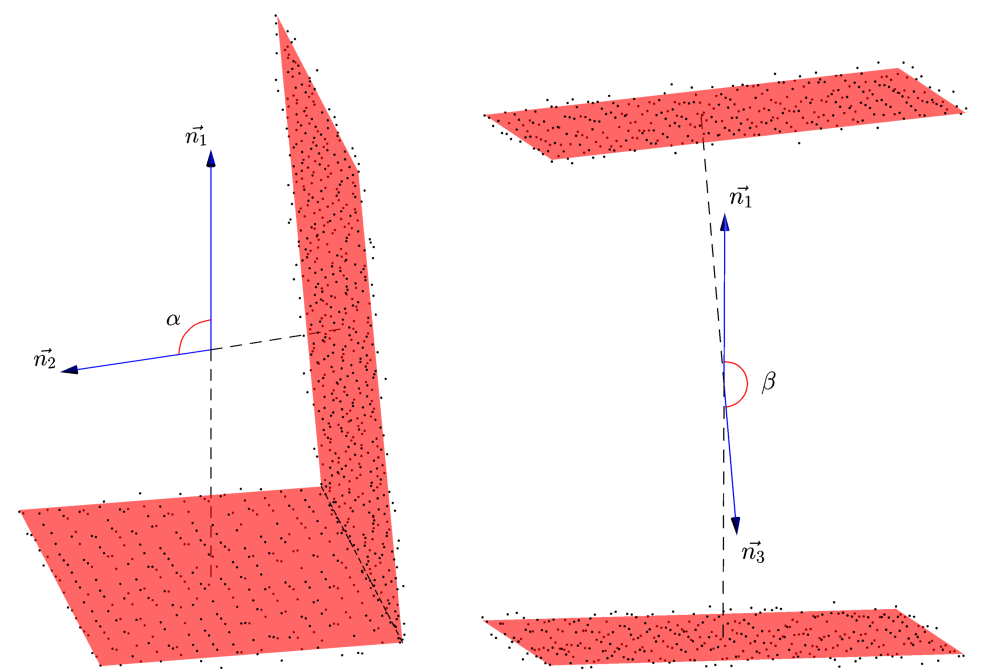

Figure 5 . The angle $\alpha$ is defined by the angle between the normal $\left(\overrightarrow{n_{1}}\right.$ and $\overrightarrow{n_{2}}$ ) of two adjacent faces which are found by fitting the cloud of points (the dots in the figures) with the equation of the best plane in the least-square sense; accordingly, $\beta$ is defined by the angle between the normal $\left(\overrightarrow{n_{1}}\right.$ and $\overrightarrow{n_{3}}$ ) of two opposite faces. For a perfect RP, $\alpha$ and $\beta$ should equal to $90^{\circ}$ and $180^{\circ}$, respectively. 
Measurement errors in resonant ultrasound spectroscopy

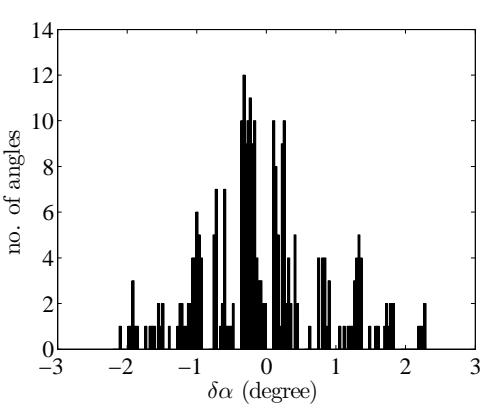

a) perpendicularity error $\delta \alpha$

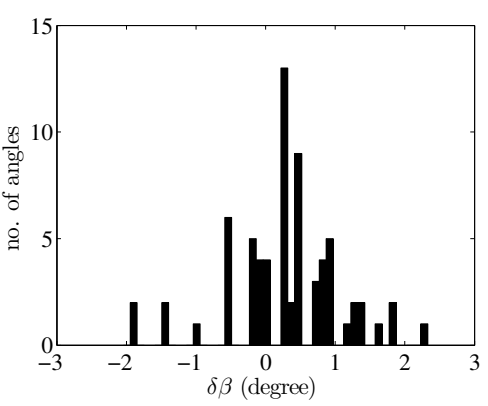

b) parallelism error $\delta \beta$

Figure 6. The distributions of the perpendicularity error $\delta \alpha$ and parallelism error $\delta \beta$ of the 23 specimens.

\section{${ }_{197}$ IV. SIMULATION OF THE ERRORS DUE TO UNCERTAINTIES ON ${ }_{198}$ RESONANT FREQUENCIES AND DIMENSIONS}

\section{A. Method}

${ }_{200}$ We consider a perfect RP specimen as a reference, characterized by the dimensions $\mathbf{d i m}^{0}$, 201 mass $m^{0}$ and stiffness constants $\mathbf{C}_{i j}^{0}$ shown in Table I. In Table I, the values of $\mathbf{d i m}^{0}$ are 202 the mean values of the dimensions of the specimens used in this work. The value of $\mathrm{m}^{0}$ was 203 calculated assuming a typical mass density value of $1.87 \mathrm{mg} / \mathrm{mm}^{3}$ taken as the mean value 204 from a former study about human femoral cortical bone ${ }^{13}$. The values of $\mathbf{C}_{i j}^{0}$ correspond to 205 the mean values of the stiffness of human femoral cortical bone at the mid-diaphysis ${ }^{13}$. The 206 first 40 eigenfrequencies $\mathbf{f}^{0}$ of the reference specimen were calculated using the RRM. This ${ }_{207}$ number of frequencies was chosen according to the experimental frequency bandwidth in RUS 208 measurements on human cortical bone specimens, which in practice contains approximately 20940 resonant frequencies.

Table I. Properties of the reference RP bone specimen. The eigenfrequencies $\mathbf{f}^{0}$ of the reference specimens are associated to the parameters in this table.

\begin{tabular}{ccc}
\hline $\operatorname{dim}^{0}(\mathrm{~mm})$ & $\mathrm{m}^{0}(\mathrm{mg})$ & $\mathbf{C}_{i j}^{0}(\mathrm{GPa})$ \\
\hline $3 \times 4 \times 5$ & 112.2 & 19.5829 .0411 .745 .834 .28 \\
\hline
\end{tabular}

${ }_{210}$ In this section, Monte-Carlo simulations ${ }^{38}$ were performed to quantify the propagation of 211 the errors due to uncertainties on resonant frequencies and specimen dimensions. Repeated 
Measurement errors in resonant ultrasound spectroscopy

212 calculations of the stiffness constants were performed, each time randomly varying the in213 put data (dimensions or/and resonant frequencies) within their stated limits of precision. ${ }_{214}$ Then we quantified the variability of each stiffness constant caused by dimension errors, by 215 frequency errors, and by the association of both dimension and frequency errors.

216 The order of magnitude of the dimension error to be used in Monte-Carlo simulations ${ }_{217}$ was obtained comparing, for each specimen, the SR- $\mu$ CT image with the dimensions dim $^{\text {exp }}$ ${ }_{218}$ measured with the caliper. Specimen's dimensions obtained from the SR- $\mu$ CT image are 219 considered as a reference based on which the uncertainty of $\mathbf{d i m}^{\text {exp }}$ can be estimated. In 220 order to obtain a representative value $\epsilon$ of the dimension error, we compared, for each 221 specimen the volume of the bone SR- $\mu \mathrm{CT}$ images and the volume of a hypothetical RP 222 of dimensions $\operatorname{dim}^{e x p} \pm \epsilon$. By equating these volumes for each of the 23 specimens and 223 solving the equations, we obtained a series of values of $\epsilon$ shown in Fig. (7). The specimen's 224 dimensions obtained from the SR- $\mu \mathrm{CT}$ image were found to be systematically smaller than ${ }_{225} \operatorname{dim}^{\exp }$. We choose the mean value of $\epsilon \approx 0.04 \mathrm{~mm}$ as a conservative value to represent the 226 accuracy of the dimensions measured by caliper. Accordingly, the uncertainty of $\operatorname{dim}^{\text {exp }}$ was 227 set to $0.04 \mathrm{~mm}$.

228 The standard error on the measured resonant frequencies used in Monte-Carlo simula229 tions was chosen to be $0.5 \%$, which is typically the repeatability of the measured resonant 230 frequencies in bones $^{17}$.

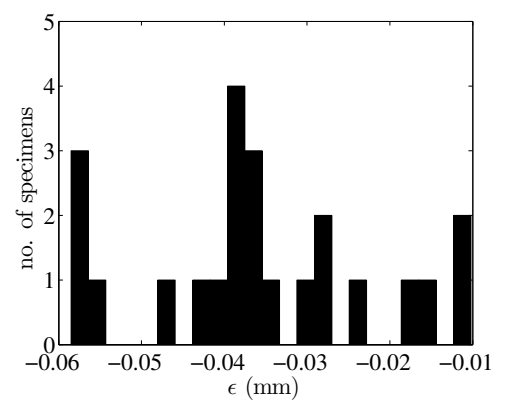

Figure 7. The distribution of the dimension error $\epsilon$ obtained by comparing for each specimen the volume of the bone SR- $\mu \mathrm{CT}$ reconstruction and the volume of a hypothetical RP of dimensions $\operatorname{dim}^{e x p} \pm \epsilon$ 
Measurement errors in resonant ultrasound spectroscopy

\section{1. Effects of uncertainties on dimension}

232

To quantify the effects of imprecise dimension measurements, 1000 random realizations of 233 dimensions were generated from independent normal distributions centered on $\mathbf{d i m}^{0}$ with a 234 standard deviation of $0.04 \mathrm{~mm}, \mathbf{d i m}^{p} \sim N\left(\mathbf{d i m}^{0}, 0.04^{2}\right)$. The number of random realizations 235 was chosen following preliminary convergence tests. For each realization $p$, the stiffness con${ }_{236}$ stants $\mathbf{C}_{i j}^{p}$, were obtained by solving the inverse problem using $\mathbf{f}^{0}$ as proxy for experimental ${ }_{237}$ frequencies, and the frequencies $\mathbf{f}^{p}$ calculated for the inadequate forward model: specimen ${ }_{238}$ of perfect RP shape with uncertain dimensions $\mathbf{d i m}^{p}$. The mass used in the forward model 239 is that of the reference RP specimen (Table I). The inverse problem uses the objective 240 function defined in Eq. (2). The stated input parameters for the simulation are summarized ${ }_{241}$ in Fig. 8 (block D).

$$
F\left(\mathbf{C}_{i j}^{p}\right)=\sum_{k}\left(\frac{f_{k}^{0}-f_{k}^{p}\left(\mathbf{C}_{i j}^{p}\right)}{f_{k}^{0}}\right)^{2}
$$
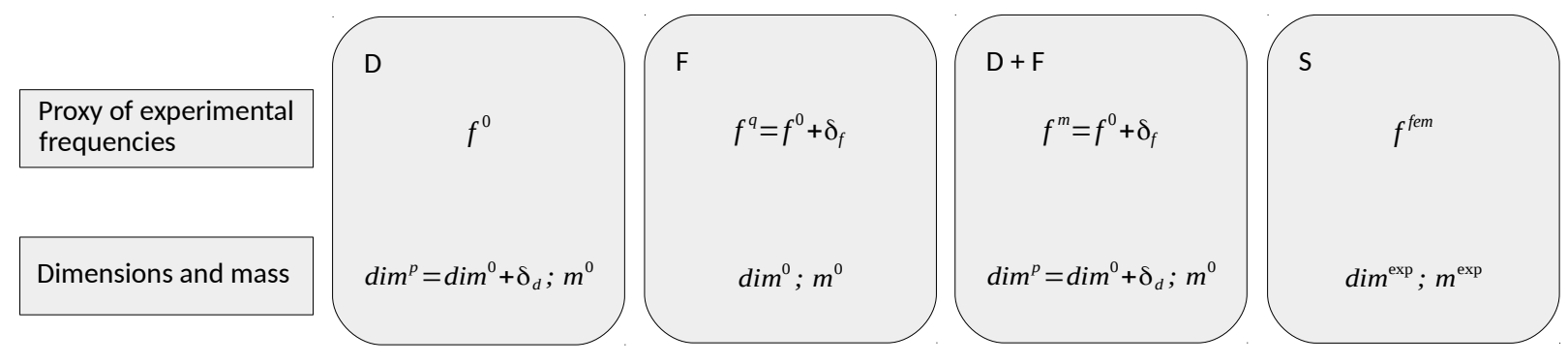

Figure 8. The input parameters for the simulations detailed in Secs. IV and V for quantifying stiffness estimation errors due to the experimental error sources: dimensions imprecision (block $\mathrm{D}$ ), frequencies imprecision (block F), dimensions and frequencies imprecision (block D $+\mathrm{F}$ ) and the imperfect specimen geometry (block S). $\delta_{d}$ and $\delta_{f}$ represent the deviations from the reference values $\operatorname{dim}^{0}$ and $f^{0}$, respectively, that are randomly generated for each realization. Given dimension and mass are the constants used for the forward model.

\section{${ }_{242}$ 2. Effects of uncertainties on frequencies}

In a similar way, for the analysis of frequency imprecision, 1000 random realizations of 244 frequencies from a normal distribution centered on $\mathbf{f}^{0}$ were generated assuming a relative ${ }_{245}$ standard deviation of $0.5 \%, \mathbf{f}^{q} \sim N\left(\mathbf{f}^{0},\left(0.005 \mathbf{f}^{0}\right)^{2}\right)$. The number of random realizations ${ }_{246}$ was chosen following preliminary convergence tests. The stiffness constants $\mathbf{C}_{i j}^{q}$, were then 
Measurement errors in resonant ultrasound spectroscopy

247 obtained by solving the inverse problem based on the objective function (Eq. (3)) using $\mathbf{f}^{q}$ 248 as proxy for experimental frequency values with an error and $\mathbf{f}^{r}$ calculated using $\operatorname{dim}^{0}, \mathrm{~m}^{0}$ 249 and assuming a perfect RP specimen (Table I). The input parameters are summarized in 250 Fig. 8 (block F).

$$
F\left(\mathbf{C}_{i j}^{q}\right)=\sum_{k}\left(\frac{f_{k}^{q}-f_{k}^{r}\left(\mathbf{C}_{i j}^{q}\right)}{f_{k}^{q}}\right)^{2}
$$

\section{3. Effects of uncertainties on dimension and frequencies}

${ }_{252}$ Finally, the effects of the association of dimension and frequency errors were analyzed 253 together. Assuming the uncertainties on frequency and dimension are $0.5 \%$ and $0.04 \mathrm{~mm}$, 254 respectively, 200 independent frequency realizations and 200 independent realizations of di255 mensions were generated from normal distributions, $\mathbf{f}^{m} \sim N\left(\mathbf{f}^{0},\left(0.005 \mathbf{f}^{0}\right)^{2}\right)$ and $\operatorname{dim}^{n} \sim$ ${ }_{256} N\left(\mathbf{d i m}^{0}, 0.04^{2}\right)$. The number of random realizations was chosen following preliminary con${ }_{257}$ vergence tests. The stiffness constants $\mathbf{C}_{i j}^{m n}$ were then obtained by solving the inverse prob${ }_{258}$ lem using $\mathbf{f}^{m}$ as proxy for experimental frequencies with errors and $\mathbf{f}^{n}$ calculated for the 259 inadequate forward model : specimen of perfect RP shape with uncertain dimensions $\mathbf{d i m}^{n}$. ${ }_{260}$ The mass used in the forward model is $\mathrm{m}^{0}$ (Table I). Precisely, this is done using the ob${ }_{261}$ jective function defined in Eq. (4). The input parameters are summarized in Fig. 8 (block $\left.{ }_{262} \mathrm{D}+\mathrm{F}\right)$.

$$
F\left(\mathbf{C}_{i j}^{m n}\right)=\sum_{k}\left(\frac{f_{k}^{m}-f_{k}^{n}\left(\mathbf{C}_{i j}^{m n}\right)}{f_{k}^{m}}\right)^{2}
$$

\section{4. Data Analysis}

${ }_{264}$ For the three cases described above, the error $\delta \mathbf{C}_{i j}^{e s t}$ is calculated for each realization of 265 the determined stiffness constants as

$$
\delta \mathbf{C}_{i j}^{e s t}=\frac{\mathbf{C}_{i j}^{e s t}-\mathbf{C}_{i j}^{0}}{\mathbf{C}_{i j}^{0}} \times 100 \%
$$

${ }_{266}$ where $\mathbf{C}_{i j}^{e s t}=\left(\mathbf{C}_{i j}^{p}, \mathbf{C}_{i j}^{q}, \mathbf{C}_{i j}^{m n}\right)$ and $\mathbf{C}_{i j}^{0}$ is the elasticity of the reference specimen. 
Measurement errors in resonant ultrasound spectroscopy

\section{${ }_{267}$ B. Results}

${ }_{268}$ The normality of the distribution of each $\delta \mathbf{C}_{i j}^{e s t}$ was verified using Shapiro-Wilk's test ${ }_{269}(p<0.05)$. Table II summarizes the distribution of $\delta \mathbf{C}_{i j}^{e s t}($ Eq. $(5))$ and the root-mean270 square error $\sigma_{f}$ representing the quality of the frequency fit at the minimum of the objective ${ }_{271}$ function. The engineering moduli, including the Young's moduli $\left(E_{1}\right.$ and $\left.E_{3}\right)$ and the ${ }_{272}$ Poisson's ratio $\left(\nu_{23}, \nu_{31}\right.$ and $\left.\nu_{21}\right)$, were also compared to the reference values (obtained from ${ }_{273} \mathbf{C}_{i j}^{0}$ in Table I). The errors are summarized in Table II. The $95 \%$ confidence intervals ${ }_{274}(\mathrm{CIs})$ of the errors were evaluated (Fig. 9). For case (D), (F) and (D+F), the 95\% CIs 275 were calculated as mean $\pm 2 \times \mathrm{SD}$. The values of the errors indicated in the following text 276 correspond to the larger absolute value of the $95 \%$ CI bounds, unless otherwise stated.

Table II. The errors (mean \pm SD in \%) on stiffness constants (Eq. (5)) and the engineering moduli due to four sources of error: uncertainties on dimension (D), on frequencies (F), on dimension and frequencies together $(\mathrm{D}+\mathrm{F})$ and imperfect specimen geometry $(\mathrm{S})$ detailed in Sec. V.

\begin{tabular}{lcccc}
\hline Error source & $\mathrm{D}$ & $\mathrm{F}$ & $\mathrm{D}+\mathrm{F}$ & $\mathrm{S}$ \\
\hline$\delta C_{11}$ & $-0.41 \pm 1.51$ & $-0.12 \pm 1.41$ & $-0.52 \pm 1.70$ & $3.56 \pm 1.61$ \\
$\delta C_{33}$ & $0.16 \pm 2.68$ & $0.00 \pm 1.44$ & $0.13 \pm 2.52$ & $-2.18 \pm 1.51$ \\
$\delta C_{13}$ & $-0.21 \pm 1.38$ & $-0.09 \pm 2.60$ & $-0.36 \pm 2.16$ & $2.22 \pm 2.08$ \\
$\delta C_{44}$ & $0.02 \pm 1.27$ & $0.00 \pm 0.53$ & $0.07 \pm 1.41$ & $-0.52 \pm 0.91$ \\
$\delta C_{66}$ & $-0.01 \pm 1.10$ & $-0.03 \pm 0.48$ & $-0.05 \pm 1.18$ & $0.85 \pm 0.68$ \\
$\delta E_{1}$ & $-0.16 \pm 1.08$ & $-0.07 \pm 0.46$ & $-0.22 \pm 1.09$ & $1.55 \pm 0.82$ \\
$\delta E_{3}$ & $0.17 \pm 2.62$ & $-0.00 \pm 1.09$ & $0.20 \pm 2.67$ & $-3.19 \pm 1.10$ \\
$\delta \nu_{23}$ & $0.03 \pm 1.92$ & $-0.01 \pm 1.94$ & $-0.06 \pm 2.68$ & $2.78 \pm 1.15$ \\
$\delta \nu_{31}$ & $0.34 \pm 2.26$ & $0.05 \pm 1.79$ & $0.32 \pm 2.24$ & $-2.01 \pm 1.53$ \\
$\delta \nu_{21}$ & $-0.50 \pm 2.06$ & $-0.14 \pm 1.89$ & $-0.55 \pm 2.24$ & $2.42 \pm 1.49$ \\
$\sigma f$ & $0.35 \pm 0.23$ & $0.43 \pm 0.06$ & $0.58 \pm 0.16$ & $0.29 \pm 0.09$ \\
\hline
\end{tabular}

277 The errors caused by dimension imprecision (case (D)) and both dimension and frequency ${ }_{278}$ imprecision (case $(\mathrm{D}+\mathrm{F})$ ) are comparable, i.e., less than $5.5 \%$ for $C_{11}, C_{33}$ and $C_{13}$, less than 
Measurement errors in resonant ultrasound spectroscopy
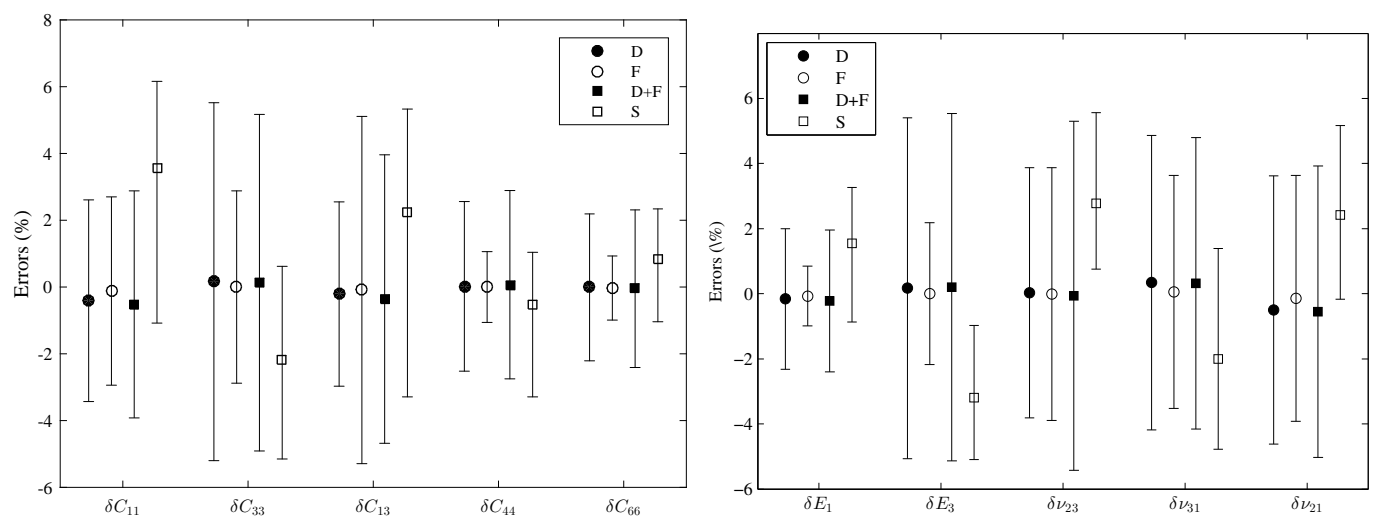

Figure 9. The mean and $95 \%$ confidence intervals of the errors on stiffness constants and engineering moduli corresponding to case (D), (F), (D+F) and (S). The error bars show the upper and lower bounds of the intervals and the mean values are represented at the center of the errorbars by the 'circle' or 'square' makers. For case $(\mathrm{D}),(\mathrm{F})$ and $(\mathrm{D}+\mathrm{F})$ the intervals were estimated as mean $\pm 2 \times \mathrm{SD}$, for case $(\mathrm{S})$ they were evaluated by fitting the cumulative distribution functions of the errors using kernel density estimators.

${ }_{279} 2.9 \%$ for $C_{44}$ and $C_{66}$. Similar observation also applies to the engineering moduli for which 280 the errors are less than $2.4 \%$ for $E_{1}, 5.5 \%$ for $E_{3}$ and $5.4 \%$ for the Poisson's ratios. Errors ${ }_{281}$ caused by frequency imprecision alone are less than $1.1 \%$ for $C_{44}$ and $C_{66}, 2.9 \%$ for $C_{11}$ and ${ }_{282} C_{33}$ and $5.3 \%$ for $C_{13}$, which agrees well with the sensitivities of resonant frequencies to the 283 stiffness constants ${ }^{29}$. The error $\delta C_{13}$ is larger when frequencies are imprecise compared to ${ }_{284}$ when dimensions are imprecise. The errors on shear stiffness constants $\left(\delta C_{44}\right.$ and $\left.\delta C_{66}\right)$ are 285 smaller than the errors on longitudinal $\left(\delta C_{11}\right.$ and $\left.\delta C_{33}\right)$ and off-diagonal stiffness constants ${ }_{286}\left(\delta C_{13}\right)$ in all the 3 cases $(\mathrm{D}, \mathrm{F}$, and $\mathrm{D}+\mathrm{F})$. Overall, $\delta E_{3}$ is two times larger than $\delta E_{1}$ and ${ }_{287}$ the accuracy associated to Young's moduli $E_{1}$ and $E_{3}$ are similar to that associated to ${ }_{288} C_{11}$ and $C_{33}$. For all the stiffness constants, $\sigma_{f}$ are around $0.35 \%, 0.43 \%$ and $0.58 \%$ when 289 dimension imprecision, frequency imprecision and both dimension and frequency imprecision 290 are considered, respectively. 
Measurement errors in resonant ultrasound spectroscopy

\section{${ }_{291}$ V. SIMULATION OF THE ERRORS DUE TO IMPERFECT SPECIMEN ${ }_{292}$ GEOMETRY}

\section{${ }_{293}$ A. Method}

294 In RUS, the inverse problem to determine stiffness constants is solved assuming that 295 the specimen is a perfect RP. In this section, we investigate the uncertainty on stiffness 296 associated to this assumption resorting to a 'virtual' RUS experiment (Fig. 8 (block S) and 297 Fig. 10):

${ }_{298}$ (1) For each of the 23 bone specimens, the resonant frequencies $\mathbf{f}^{\text {fem }}$ were calculated using 299 the finite element method considering the actual specimen's geometry derived from SR- $\mu \mathrm{CT}$ 300 images, measured mass $m^{e x p}$ and specimen's stiffness $\mathbf{C}_{i j}^{e x p}$ determined in the usual manner 301 assuming a perfect RP shape. Details on the finite element implementation are given in 302 appendix (Appendix A).

${ }_{303}$ (2) The stiffness constants $\mathbf{C}_{i j}^{f e m}$ of each specimen were estimated solving the inverse 304 problem defined by the frequencies $\mathbf{f}^{\text {fem }}$ (the first 40 frequencies) considered as measurements 305 and a forward model characterized by a perfect RP geometry (dimensions dim $^{\text {exp }}$ ) and 306 specimen's mass (mexp) (Sec. III).

307 These resulting $\mathbf{C}_{i j}^{f e m}$ are the stiffness constants of a RP bone specimen that would exhibit 308 the same resonant frequencies as the imperfect shape bone specimens with stiffness constants ${ }_{309} \mathbf{C}_{i j}^{e x p}$. Constants $\mathbf{C}_{i j}^{f e m}$ are biased by imperfect specimen geometry and are compared to the 310 true stiffness constants of the specimen used in the FEM model $\left(\mathbf{C}_{i j}^{e x p}\right)$. Namely, we calculate 311 the errors $\delta \mathbf{C}_{i j}^{f e m}=\frac{\mathbf{C}_{i j}^{f e m}-\mathbf{C}_{i j}^{e x p}}{\mathbf{C}_{i j}^{e x p}} \times 100 \%$.

\section{B. Results}

313 The errors on stiffness constants and the engineering moduli due to imperfect geometry 314 of the specimens are summarized in Table II (last column). As only 23 specimens were 315 included and the errors were not normally distributed, the 95\% CIs of the errors (Table III 316 and Fig. 9) were evaluated by fitting the cumulative distribution functions of the errors 317 using kernel density estimators. For all the stiffness constants, there is a bias, i.e. the 318 mean value of the errors is not zero and it can be positive or negative depending on the 319 constant (the mean values vary from $-3.19 \%$ to $3.56 \%$ ). The SD of the errors varies from 
Measurement errors in resonant ultrasound spectroscopy

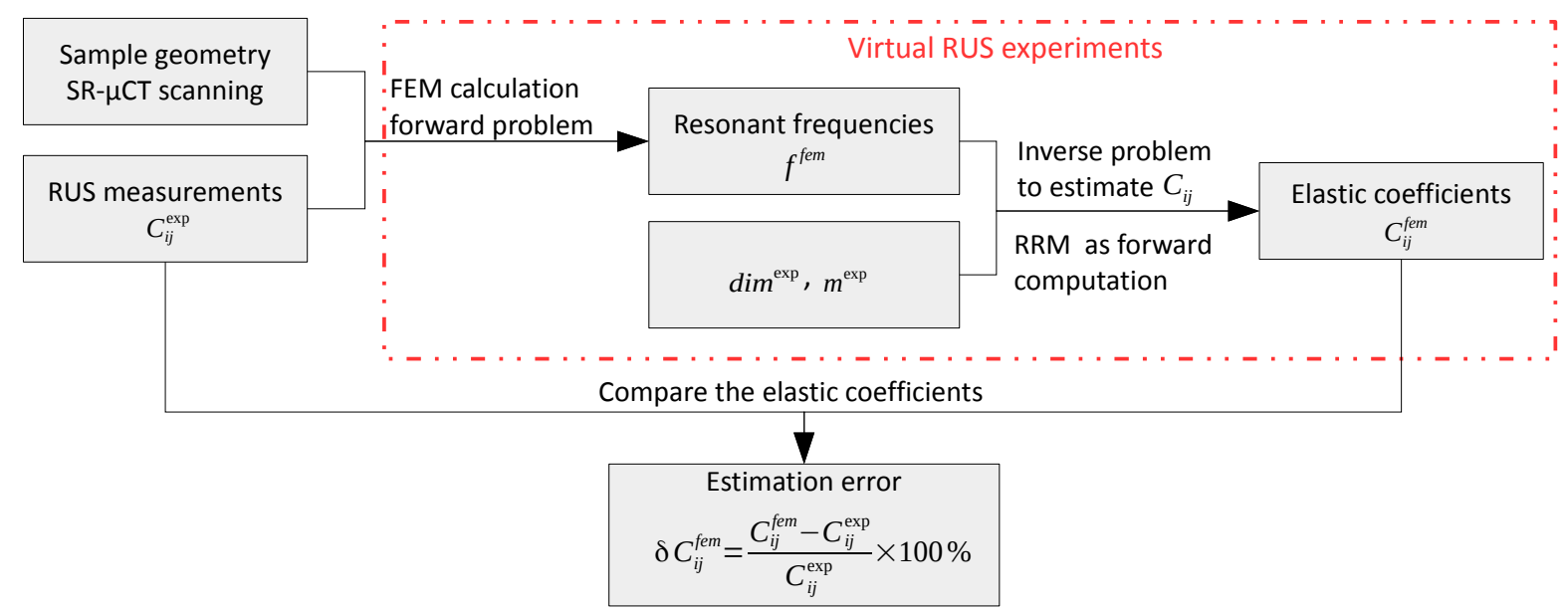

Figure 10. Diagram of the FEM simulation for quantifying the bias caused by imperfect specimen geometry. $\mathbf{C}_{i j}^{e x p}$ are bone stiffness constants measured by RUS (Section III B), $\mathbf{f}^{\text {fem }}$ are the resonant frequencies calculated from the actual specimen geometry, $\operatorname{dim}^{e x p}$ are the dimensions of the specimens measured by caliper, $\mathbf{C}_{i j}^{f e m}$ are the stiffness constants calculated by solving the inverse problem, and $\delta \mathbf{C}_{i j}^{f e m}$ represent the estimation errors.

$3200.68 \%$ to $2.08 \%$. In particular, the errors on shear stiffness constants present a smaller 321 variation than longitudinal and off-diagonal ones (see the 95\% CIs in Table III) and the errors 322 on Young's moduli present slightly less variability compared to the longitudinal stiffness 323 constants (Table II and III).

Table III. The $95 \%$ CIs (in \%) of the errors on stiffness constants and the engineering moduli due to imperfect specimen geometry.

\begin{tabular}{cccccc}
\hline & $\delta C_{11}$ & $\delta C_{33}$ & $\delta C_{13}$ & $\delta C_{44}$ & $\delta C_{66}$ \\
\hline $95 \%$ CI & {$[-1.08,6.16]$} & {$[-5.15,0.62]$} & {$[-3.29,5.33]$} & {$[-3.29,1.04]$} & {$[-1.04,2.34]$} \\
\hline$\delta E_{1}$ & $\delta E_{3}$ & $\delta \nu_{23}$ & $\delta \nu_{31}$ & $\delta \nu_{21}$ \\
\hline $95 \%$ CI & {$[-0.87,3.27]$} & {$[-5.09,-0.98]$} & {$[0.75,5.56]$} & {$[-4.78,1.39]$} & {$[-0.17,5.17]$} \\
\hline
\end{tabular}

\section{VI. DISCUSSION AND CONCLUSION}

325 In this study, we performed simulations to quantify the errors on the stiffness constants 326 determined from RUS measurements. We used typical elasticity values of human cortical ${ }_{327}$ bone as reference and studied the effects of errors due to (1) uncertainties on the mea- 
Measurement errors in resonant ultrasound spectroscopy

328 surement of frequencies; (2) uncertainties on the measurement of dimensions (assuming a ${ }_{329}$ perfect RP shape); (3) imperfect specimen's geometry (deviation from a perfect RP). The 330 first two points were addressed with a calculation of error propagation with Monte-Carlo 331 simulations which require a statistical model of the quantities investigated. For dimensions 332 (of an assumed perfect RP) and frequencies, it is reasonable to assume normal distributions 333 around the reference values. The third source of error is the deviation of the shape from a ${ }_{334}$ perfect RP. In that case we do not have a statistical model for the shape alterations, i.e., 335 Monte-Carlo simulations cannot be used. Hence, the third point was addressed using actual 336 experimental data on a collection of 23 bone specimens. The main parameters of the Monte${ }_{337}$ Carlo simulations were the assumed level of error on experimentally determined resonant 338 frequencies, set to $0.5 \%$, and experimentally determined specimen's dimensions, set to 0.04 $339 \mathrm{~mm}(\sim 1 \%)$. The choice of these values is consistent with our experience of using RUS to 340 measure bone specimens ${ }^{17}$.

${ }_{341}$ Using micro-CT, we could quantify the range of geometrical errors associated to a simple 342 specimen's preparation procedure. We found that perpendicularity and parallelism errors ${ }_{343}$ were in average less than $1^{\circ}$ and always less than $2^{\circ}$ (Fig. 6).

344 Overall, we found errors on elasticity values of a few percents, or less than one percent, 345 depending on the considered stiffness constant. Note that we discuss the accuracy errors 346 reporting the $95 \%$ CIs of the error. Consistent with the findings of several previous stud${ }_{347}$ ies $^{26,29}$, we found that the off-diagonal stiffness constants presented the highest errors and 348 shear constants the smallest ones. This is related to the higher sensitivity of RUS to shear 349 stiffness constants. Comparing the uncertainties of the sources of error (dimensions and 350 frequencies) and the uncertainties of the errors on shear stiffness constants (the most pre351 cisely determined ones), comparable values were observed (Table II), i.e., $0.04 \mathrm{~mm}(\sim 1 \%)$ 352 uncertainty on dimensions and $0.5 \%$ uncertainty on frequencies leads to $\sim 1.2 \%$ and $\sim 0.5 \%$ 353 uncertainties on the errors of shear stiffness constants, respectively. Additional Monte-Carlo 354 simulations, following the same routine in Section IV A showed that increasing the error level 355 of dimensions and frequencies by $20 \%$, i.e., the uncertainties of dimensions and frequencies 356 became $0.05 \mathrm{~mm}$ and $0.6 \%$, respectively, will increase the CIs of the error on shear stiffness 357 by $\sim 16 \%$ to $25 \%$, approximately.

${ }_{358}$ For all the stiffness constants but $C_{13}$, dimension uncertainties lead to larger errors in 359 elasticity compared to the case where only frequency uncertainties are considered (Table II 
Measurement errors in resonant ultrasound spectroscopy

360 and Fig. 9). For $C_{13}$, the largest error is observed for frequency uncertainties, suggesting that ${ }_{361} C_{13}$ may be less sensitive to dimension imperfections than to resonant frequencies in current 362 simulation conditions. Interestingly, dimension uncertainties or a coupling of frequency and 363 dimension uncertainties caused similar levels of errors on the stiffness constants (Table II 364 and Fig. 9).

Deviation of the actual specimen's shape from a perfect RP affects the accuracy of the 366 stiffness constants measured with RUS. This is because the forward model used to solve the 367 inverse problem, assuming a perfect RP geometry, is not correct. The approach introduced 368 in Section V aimed at simulating the effect of this source of error. It is important to 369 note that, in general (when a micro-CT scan of the specimen is not available), only the 370 mass can be accurately measured as opposed to the dimensions (because the geometry is 371 in general not perfect). This is the reason why mass $\left(\mathrm{m}^{e x p}\right)$ but not mass density was used 372 in the simulations in Secs. IV and V. The uncertainty of the mass was about $0.1 \%$ since 373 the precision of the balance is $\pm 0.1 \mathrm{mg}$ and the mass of the bone specimens are around $374100 \mathrm{mg}$. A linear relationship exists between mass and stiffness constants, consequently, 375 for given dimensions and resonant frequencies ${ }^{26}$, a mass uncertainty of $0.1 \%$ will cause the 376 same uncertainty $(0.1 \%)$ on the stiffness constants, which is negligible compared to the error 377 levels caused by other factors. Accordingly, the uncertainty of mass was not considered in 378 this work. We have observed that most of the caliper-measured volumes were overestimated 379 of approximately $3 \%$ in average compared to the volumes deduced from SR- $\mu \mathrm{CT}$ images. 380 Accordingly, the quantified elasticity errors are a result of both overestimated dimensions 381 and irregularity of the RP shape. The elasticity errors due to an imperfect RP geometry ${ }_{382}$ (Table III and Fig 9) were between 2.3\% 6.2\%. The comparison of the contribution of the 383 three sources of errors to the precision of RUS measurements shows that errors due to an 384 imperfect geometry are found to be of the same order as the errors calculated by Monte-Carlo 385 simulations caused by frequency or dimension uncertainties in our specific case.

386 It is noteworthy that the values of $\sigma_{f}$ obtained from simulations in the present study ${ }_{387}\left(\sigma_{f} \approx 0.58 \%\right.$ with Monte-Carlo simulations and $\sigma_{f} \approx 0.29 \%$ using FEM simulations with 388 the imperfect shape) are similar to values reported for actual RUS measurements of bone 389 and other attenuative materials ${ }^{17,22}$ where $\sigma_{f}$ is typically in the range $0.25-0.40 \%$. This 390 suggests that the simulations accurately reproduce the experimental error characteristic of 391 RUS measurements. The level of errors quantified in the present study are consistent with 
Measurement errors in resonant ultrasound spectroscopy

392 the reported precision of RUS for human cortical bone application (3\%, $5 \%$ and $0.4 \%$ for 393 longitudinal, off-diagonal and shear stiffness constants, respectively $)^{17}$, estimated from the ${ }_{394} \operatorname{RMSE} \sigma_{f}$.

395 This study has introduced an original methodology to quantify errors in RUS measure396 ments. The method was applied to bone but could be used to assess the accuracy for RUS 397 measurements of various materials. Note that it has been possible to implement Monte${ }_{398}$ Carlo simulations only because an automated pairing of frequencies (for the calculation of 399 the objective function) was possible. This automated pairing was initially developed to ${ }_{400}$ process spectra of attenuative materials where several resonant peaks can not be retrieved ${ }^{34}$ 401 and it is also efficient to process synthetic resonant frequencies as in the present study where 402 no peak is missing.

${ }_{403}$ In RUS measurements, specimens are assumed to be homogeneous, although cortical 404 bone specimens are inhomogeneous to some extent. When the wavelength is much greater 405 than the length scale of the inhomogeneity, the material can be regarded as a homogeneous 406 material. A conservative estimation of acceptable inhomogeneity in RUS was suggested by ${ }_{407}$ Ulrich et.al. ${ }^{28}$. The maximum size of an inhomogeneity should be smaller than a threshold ${ }_{408} \xi \leq 2 l / n$, where $l$ is the smallest dimension of the sample and $n$ can be taken as the number 409 of the considered resonant frequencies. Here with $l=3 \mathrm{~mm}$ and $n=40$, the threshold ${ }_{410}$ is $\xi=150 \mu \mathrm{m}$, which is larger than the diameter of the pores in human cortical bone ${ }_{411}$ (Haversian canals diameter is typically in the range of $20-100 \mu \mathrm{m}$ ). According to this ${ }_{412}$ criterion, bone specimens in the present work may be considered as homogeneous.

${ }_{413}$ Aside of the uncertainties in the values of the inputs in RRM, including mass, dimensions ${ }_{414}$ and stiffness constants, the RRM has a limited accuracy associated to the truncation to M415 th order of the polynomial approximation of the displacement field. Resonant frequencies ${ }_{416}$ calculated with RRM are more accurate with increasing values of $\mathrm{M}$ but as a counterpart, ${ }_{417}$ the computing time increases. In the present work, this was a critical issue because large 418 numbers of iterations were involved to solve the inverse problem in the Bayesian framework. ${ }_{419}$ In practice, $M=10$ used in this study, following the suggestion by Migliori and Sarrao ${ }^{26}$, is a 420 good compromise between accuracy and computing time if the first 50 resonant frequencies 421 are considered. A preliminary test showed that the root-mean-square-error between the ${ }_{422}$ RRM-yielded frequencies when $M=10$ and $M=20$ is close to $0.07 \%$ for the first 40 ${ }_{423}$ frequencies, which is negligible compared to the magnitude of other sources of error that we 
Measurement errors in resonant ultrasound spectroscopy

424 handled with in this work.

${ }_{425}$ This study has some limitations. We used simulated resonant frequencies as proxy for ${ }_{426}$ RUS data as input to the inverse problem. Precisely, the eigenfrequencies of the first forty ${ }_{427}$ vibration modes were used. In actual RUS experiments to measure bone, a maximum of ${ }_{428}$ fifteen to twenty frequencies among the first forty can actually be retrieved due to peak ${ }_{429}$ overlapping $^{22}$. In theory, taking into account more frequencies should improve the precision 430 of the determination of stiffness constants because more information is used for the inverse ${ }_{431}$ problem. However, in practice, the achievable precision also depends on the quality of the ${ }_{432}$ frequency measurement which decreases in the higer frequency range due to the increased 433 modal density and peak overlapping. Since the resonant frequencies are much more sensitive ${ }_{434}$ to shear stiffness constants ${ }^{39}$, it is expected that using less frequencies than in the present ${ }_{435}$ study would essentially decrease the precision of constants $C_{11}, C_{33}$ and $C_{13}$ but would have ${ }_{436}$ little impact on the precision of the shear stiffness constants. The results of the simulation ${ }_{437}$ in Sec. V critically rely on the actual pixel size in SR- $\mu$ CT experiments, because the exact ${ }_{438}$ shape of the specimens were used to compute the 'true' resonant frequencies for the inverse 439 problem. However, we did not perform calibration for identifying the actual pixel size during ${ }_{440} \mathrm{SR}-\mu \mathrm{CT}$ experiments. This could partly affect or bias the results. Another limitation is ${ }_{441}$ that we did not simulate the error on stiffness constants due to a combination of frequency ${ }_{442}$ uncertainty and imperfect RP geometry. In view of the results of Sec. IV, we expect that ${ }_{443}$ elasticity errors would only be slightly larger. Furthermore, some sources of errors in RUS ${ }_{444}$ have not been considered such as the effect of imperfect boundary conditions ${ }^{40}$ and the 445 uncertainty on the measurement of specimen's mass.

${ }_{446}$ The validation of the measurement of bone elasticity with RUS relies (1) on the successful ${ }_{447}$ measurement of a reference transverse isotropic material with a Q-factor similar as bone's ${ }_{448} \mathrm{Q}_{\text {-factor }}{ }^{22}$; (2) on the comparison of the stiffness constants obtained with RUS and from ${ }_{449}$ the independent measurement of the time-of-flight of shear and longitudinal waves in bone 450 specimens ${ }^{16,17}$; and (3) on the results of the present study focused on the quantification of ${ }_{451}$ accuracy errors. The latter suggest that despite the typical non-perfect geometry of bone ${ }_{452}$ specimens and despite the relatively large uncertainty in the measurement of the bone reso${ }_{453}$ nance frequencies (due to attenuation), the stiffness constants are obtained with a maximum ${ }_{454}$ error of a few percents. A very conservative accuracy value can be quantified by the larger 455 absolute value of the (non symetric) 95\% CI bounds; accuracy defined like this was $6.2 \%$ 
Measurement errors in resonant ultrasound spectroscopy

${ }_{456}$ for longitudinal stiffness and 3.3\% for shear stiffness, $5.1 \%$ for Young's moduli and 5.6\% for ${ }_{457}$ Poisson's ratios (Table III).

${ }_{458}$ To further enhance the accuracy of bone RUS measurement, possible paths would be 459 (1) using a specific implementation of the Rayleigh-Ritz method for nonrectangular par${ }_{460}$ allelepiped specimen ${ }^{29}$, provided that the angles between the specimen's surfaces can be 461 measured; (2) decreasing the frequency uncertainty by improving the signal processing of ${ }_{462}$ RUS spectra.

\section{${ }_{463}$ ACKNOWLEDGMENTS}

${ }_{464}$ This work has received financial support from the Agency National Research under the 465 ANR-13-BS09-0006 MULTIPS and ANR-14-CE35-0030-01 TaCo-Sound projects and was ${ }_{466}$ done in the framework of LabEx PRIMES (ANR-11-LABX-0063) of Université de Lyon. ${ }_{467}$ The authors would like to thank Rémy Gauthier, Hélène Follet and David Mitton for the 468 collection of bone specimens and the help in conducting SR- $\mu \mathrm{CT}$ imaging experiments. The 469 authors wish to acknowledge Didier Cassereau for providing technical support with numerical 470 computations and Pascal Dargent for designing the setup and the protocol of bone specimen 471 preparation, as well as the ESRF for providing beamtime through the experiment MD927 472 and Lukas Helfen for his assistance in image acquisition on beamline ID19.

\section{${ }_{473}$ Appendix A: Calculation of resonant frequencies using Finite element 474 modeling (FEM)}

475 Bone was modeled as a homogeneous transversely isotropic material. The bone volumes 476 obtained from the SR- $\mu \mathrm{CT}$ were discretized into about 3 million quadratic tetrahedral el477 ements. This corresponded to a maximum element size of $0.12 \mathrm{~mm}$, which was chosen 478 after a convergence study and ensures at least 10 elements per smallest wavelength in the 479 investigated frequency bandwidth. A modal analysis was conducted to calculate the eigen480 frequencies. We used the software Code-Aster (ver 12.5, EDF R\&D, France, license GNU ${ }_{481}$ GPL, http://www.code-aster.org).

${ }_{482}$ The accuracy of the finite element model was evaluated by comparing the first $40 \mathrm{FEM}$ 483 eigeinfrequencies to eigenfrequencies calculated with the Rayleigh-Ritz method for a perfect 
${ }_{484} \mathrm{RP}$ bone specimen (Table I). The RMSE $\sigma_{f}$ between eigenfrequencies calculated by the two ${ }_{485}$ methods was $\sim 0.06 \%$. After solving the inverse problem using FEM eigenfrequencies, the ${ }_{486}$ errors in the stiffness constants were $\sim 0.05 \%, 0.60 \%$ and $0.30 \%$ on shear, longitudinal and 487 off-diagonal stiffness constants. These errors are at least one order of magnitude smaller 488 than the errors related to shape imperfections (Sec. V).

\section{${ }_{489}$ REFERENCES}

${ }_{490}{ }^{1}$ J. Klein-Nulend, P. J. Nijweide, and E. H. Burger, "Osteocyte and bone structure," 491 Current osteoporosis reports 1, 5-10 (2003).

${ }_{492}^{2}$ P. Fratzl and R. Weinkamer, "Natures hierarchical materials," Progress in Materials Sci493 ence 52, 1263-1334 (2007).

${ }_{494}^{3}$ J.-Y. Rho, L. Kuhn-Spearing, and P. Zioupos, "Mechanical properties and the hierarchical 495 structure of bone," Medical engineering \& physics 20, 92-102 (1998).

${ }_{496}{ }^{4}$ Q. Grimal, K. Raum, A. Gerisch, and P. Laugier, "A determination of the minimum sizes of representative volume elements for the prediction of cortical bone elastic properties," Biomechanics and modeling in mechanobiology 10, 925-937 (2011).

${ }_{499}^{5}$ J. D. Currey, Bones: structure and mechanics (Princeton university press, Princeton, 500 2002), pp. 436.

${ }_{501}^{6}$ D. Rohrbach, Q. Grimal, P. Varga, F. Peyrin, M. Langer, P. Laugier, and K. Raum, ${ }_{502}$ "Distribution of mesoscale elastic properties and mass density in the human femoral shaft," ${ }_{503}$ Connective tissue research 56, 120-132 (2015).

${ }_{504}^{7}{ }^{7}$ S. B. Lang, "Elastic coefficients of animal bone," Science 165, 287-288 (1969).

${ }_{505}^{8}$ J. L. Katz and H. S. Yoon, "The structure and anisotropic mechanical properties of bone," 506 IEEE Trans. Biomed. Eng. BME-31, 878 -884 (1984).

${ }_{507}^{9}$ R. Ashman, J. Rho, and C. Turner, "Anatomical variation of orthotropic elastic moduli 508 of the proximal human tibia," Journal of biomechanics 22, 895-900 (1989).

${ }_{509}^{10} \mathrm{~J}$. Y. Rho, "An ultrasonic method for measuring the elastic properties of human tibial 510 cortical and cancellous bone." Ultrasonics 34, 777-783 (1996).

${ }^{511}{ }^{11} \mathrm{C}$. Schwartz-Dabney and P. Dechow, "Accuracy of elastic property measurement in 512 mandibular cortical bone is improved by using cylindrical specimens," Journal of biome513 chanical engineering 124, 714-723 (2002). 
${ }_{514}^{12}$ A. A. E. Orías, J. M. Deuerling, M. D. Landrigan, J. E. Renaud, and R. K. Roeder, 515 "Anatomic variation in the elastic anisotropy of cortical bone tissue in the human femur," 516 Journal of the mechanical behavior of biomedical materials 2, 255-263 (2009).

${ }_{517}{ }^{13}$ M. Granke, Q. Grimal, A. Saed, P. Nauleau, F. Peyrin, and P. Laugier, "Change in porosity 518 is the major determinant of the variation of cortical bone elasticity at the millimeter scale 519 in aged women," Bone 49, 1020-1026 (2011).

${ }_{520}^{14}$ E. Lefèvre, P. Lasaygues, C. Baron, C. Payan, F. Launay, H. Follet, and M. Pithioux, "An${ }_{521}$ alyzing the anisotropic hooke s law for children s cortical bone," Journal of the mechanical 522 behavior of biomedical materials 49, 370-377 (2015).

${ }_{523}{ }^{15}$ R. G. Leisure and F. Willis, "Resonant ultrasound spectroscopy," Journal of Physics: 524 Condensed Matter 9, 6001 (1997).

${ }_{525}^{16}$ L. Peralta, X. Cai, P. Laugier, and Q. Grimal, "A critical assessment of the in-vitro 526 measurement of cortical bone stiffness with ultrasound." Ultrasonics 80, 119-126 (2017). ${ }_{527}^{17}$ S. Bernard, Q. Grimal, and P. Laugier, "Accurate measurement of cortical bone elasticity ${ }_{528}$ tensor with resonant ultrasound spectroscopy," Journal of the mechanical behavior of ${ }_{529}$ biomedical materials 18, 12-19 (2013).

${ }_{530}{ }^{18}$ T. Delaunay, E. L. Clezio, M. Guennou, H. Dammak, M. P. Thi, and G. Feuillard, ${ }_{531}$ "Full tensorial characterization of pzn-12\%pt single crystal by resonant ultrasound spec532 troscopy," and Frequency Control IEEE Transactions on Ultrasonics, Ferroelectrics 55, $533 \quad 476-488$ (2008).

${ }_{534}^{19} \mathrm{R}$. Schwarz and J. Vuorinen, "Resonant ultrasound spectroscopy: applications, current 535 status and limitations," Journal of Alloys and Compounds 310, 243-250 (2000).

${ }_{536}{ }^{20} \mathrm{~W}$. H. Wang, "The elastic properties, elastic models and elastic perspectives of metallic ${ }_{537}$ glasses," Progress in Materials Science 57, 487-656 (2012).

${ }_{538}^{21}$ H. Ledbetter, C. Fortunko, and P. Heyliger, "Orthotropic elastic constants of a boron539 aluminum fiber-reinforced composite: An acoustic-resonance-spectroscopy study," Journal 540 of Applied Physics 78, 1542-1546 (1995).

${ }_{541}^{22}$ S. Bernard, Q. Grimal, and P. Laugier, "Resonant ultrasound spectroscopy for viscoelastic ${ }_{542}$ characterization of anisotropic attenuative solid materials," The Journal of the Acoustical 543 Society of America 135, 2601-2613 (2014).

${ }_{544}^{23}$ R. Longo, T. Delaunay, D. Laux, M. El Mouridi, O. Arnould, and E. Le Clezio, "Wood 545 elastic characterization from a single sample by resonant ultrasound spectroscopy," Ultra- 
sonics 52, 971-974 (2012).

${ }_{547}^{24}$ T. Lee, R. S. Lakes, and A. Lal, "Investigation of bovine bone by resonant ultrasound 548 spectroscopy and transmission ultrasound," Biomechan. Model. Mechanobiol. 1, 165-175 549 (2002).

${ }_{550}^{25}$ J. H. Kinney, J. R. Gladden, G. W. Marshall, S. J. Marshall, J. H. So, and J. D. May551 nard, "Resonant ultrasound spectroscopy measurements of the elastic constants of human 552 dentin." J Biomech 37, 437-441 (2004).

${ }_{553}^{26}$ A. Migliori and J. L. Sarrao, Resonant Ultrasound Spectroscopy (Wiley, New York, 1997), $554 \quad$ pp. 202.

${ }_{555}^{27}$ A. Migliori and J. D. Maynard, "Implementation of a modern resonant ultrasound spec556 troscopy system for the measurement of the elastic moduli of small solid specimens," Rev. 557 Sci. Instrum. 76, 121301 (2005).

${ }_{558}{ }^{28}$ T. J. Ulrich, K. McCall, and R. Guyer, "Determination of elastic moduli of rock samples 559 using resonant ultrasound spectroscopy," The Journal of the Acoustical Society of America 560 111, 1667-1674 (2002).

${ }_{561}^{29}$ M. Landa, P. Sedlák, H. Seiner, L. Heller, L. Bicanová, P. Šittner, and V. Novák, "Modal 562 resonant ultrasound spectroscopy for ferroelastics," Appl. Phys., A 96, 557-567 (2009). ${ }_{563}^{30}$ P. Sedlák, H. Seiner, J. Zídek, M. Janovská, and M. Landa, "Determination of all 21 564 independent elastic coefficients of generally anisotropic solids by resonant ultrasound spec565 troscopy: Benchmark examples," Experimental Mechanics 54, 1073-1085 (2014).

${ }_{566}^{31}$ A. Migliori, J. L. Sarrao, W. M. Visscher, T. M. Bell, M. Lei, Z. Fisk, and R. G. Leisure, ${ }_{567}$ "Resonant ultrasound spectroscopic techniques for measurement of the elastic moduli of 568 solids," Physica B 183, 1 - 24 (1993).

${ }_{569}^{32}$ A. Lebedev, L. Ostrovskii, A. Sutin, I. Soustova, and P. Johnson, "Resonant acoustic 570 spectroscopy at low q factors," Acoustical Physics 49, 81-87 (2003).

${ }^{571}{ }^{33}$ S. Bernard, J. Schneider, P. Varga, P. Laugier, K. Raum, and Q. Grimal, "Elasticity572 density and viscoelasticity-density relationships at the tibia mid-diaphysis assessed from 573 resonant ultrasound spectroscopy measurements." Biomech Model Mechanobiol 15, 97-109 574 (2016).

${ }^{575}{ }^{34}$ S. Bernard, G. Marrelec, P. Laugier, and Q. Grimal, "Bayesian normal modes identifi576 cation and estimation of elastic coefficients in resonant ultrasound spectroscopy," Inverse 577 Problems 31, 065010 (2015). 
${ }_{578}^{35}$ H. S. Yoon and J. L. Katz, "Ultrasonic wave propagation in human cortical boneii. mea579 surements of elastic properties and microhardness," Journal of biomechanics 9, 459-464 580 (1976).

${ }_{581}^{36}$ M. Salomé, F. Peyrin, P. Cloetens, C. Odet, A. M. Laval-Jeantet, J. Baruchel, and ${ }_{582}$ P. Spanne, "A synchrotron radiation microtomography system for the analysis of trabec583 ular bone samples," Medical Physics 26, 2194-2204 (1999).

${ }_{584}^{37}$ T. Weitkamp, P. Tafforeau, E. Boller, P. Cloetens, J.-P. Valade, P. Bernard, F. Peyrin, ${ }_{585}$ W. Ludwig, L. Helfen, and J. Baruchel, "Status and evolution of the esrf beamline id19," ${ }_{586}$ in X-ray Optics and Microanalysis: Proceedings of the 20th International Congress, Vol. $587 \quad 1221$ (2010) pp. 33-38.

${ }_{588}^{38} \mathrm{G}$. Anderson, "Error propagation by the monte carlo method in geochemical calculations," 589 Geochimica et Cosmochimica Acta 40, 1533-1538 (1976).

${ }_{590}^{39}$ B. J. Zadler, J. H. Le Rousseau, J. A. Scales, and M. L. Smith, "Resonant ultrasound 591 spectroscopy: theory and application," Geophysical Journal International 156, 154-169 592 (2004).

${ }_{593}^{40} \mathrm{~A}$. Yoneda, "Intrinsic eigenvibration frequency in the resonant ultrasound spectroscopy," 594 Earth, planets and space 54, 763-770 (2002). 